\title{
An Experimental Comparison of Linear and Parabolic Tapered Waveguide Lasers and a Demonstration of Broad-Stripe Diode Pumping
}

\author{
S. J. Hettrick, J. Wang, C. Li, J. S. Wilkinson, and D. P. Shepherd
}

\begin{abstract}
This paper compares the laser performance of linear and parabolic tapered waveguides in ion-exchanged Nd:Glass, finding significant advantage for the linear guides, with demonstrated adiabatic expansion to widths of $250 \mu \mathrm{m}$. The characterization of these waveguide lasers by Ti:sapphire pumping is followed by a demonstration of high-power diode-pumped laser operation and a discussion of the optimization of such a scheme for producing high-power integrated-optics laser sources.
\end{abstract}

Index Terms-Ion exchange, tapers, waveguide lasers.

\section{INTRODUCTION}

$\mathbf{S}$ CALING the average power of diode-pumped integratedoptics laser sources requires the use of multimode waveguides to enable pumping by nondiffraction-limited high-power diode sources. However, a standard specification for the integrated laser is operation in the fundamental spatial mode. One method of satisfying these opposing requirements is through the use of a structure that has a taper connecting a multimode broadstripe section to a single-mode channel section of the waveguide [1]-[3]. The taper must be designed such that the fundamental mode, propagating from the channel to the broad-stripe, suffers minimal coupling to higher order modes, as this power would be lost from the laser resonator when it returns through the taper to the channel.

Linear tapered waveguide structures have been previously applied to diode laser sources themselves in order to increase the mode size and thereby avoid facet damage for high-power operation [4]. Here, our design consideration for the maximum width of the taper $W_{\max }$ is that we should be able to confine the nondiffraction-limited slow axis of a broad-stripe diode pump laser, which may typically have an $M^{2}$ value of up to 40 . As a first approximation we can say that this consideration can be quantified as

$$
W_{\max } \geq \frac{\lambda_{p} M^{2}}{\mathrm{NA}}
$$

where $\lambda_{p}$ is the diode wavelength and NA is the numerical aperture of the waveguide (i.e., we want the waveguide NA to be greater than the divergence of the pumping beam). For the ion-exchanged waveguides considered here the maximum index change is $8 \times 10^{-3}$ for the TE polarization [5] and so tapers

Manuscript received June 30, 2003; revised November 24, 2003. This work was supported by the U.K. EPSRC under Grant GR/M83964/01.

The authors are with the Optoelectronics Research Centre, University of Southampton, Highfield, Southampton, U.K. (e-mail: dps@orc.soton.ac.uk).

Digital Object Identifier 10.1109/JLT.2004.824548 up to a maximum width of $\sim 200 \mu \mathrm{m}$ are required. Various designs for the shape of the taper have been proposed for passive mode-expanding devices, including linear, parabolic, and exponential expansions, and it has been suggested that considerations of diffraction and ray tracing lead to a preference for the parabolic taper shape, which has a uniform mode-coupling constant along its length [6]. A design rule for the length $l_{\text {tap }}$ of an adiabatic parabolic taper, based upon keeping the side-wall expansion angle at a given point $\theta_{p}(z)$ less than the diffraction spreading of the lowest order mode at that point $z$, was given by Milton and Burns as [6]

$$
l_{\mathrm{tap}} \approx \frac{n W_{\max }^{2}}{2 \alpha \lambda_{s}}
$$

where $\lambda_{s}$ is the laser wavelength, $n$ is the mode effective index, and $\alpha$ is the taper expansion coefficient. Adiabatic expansion is expected to occur for $\alpha \leq 1$ and so taper lengths of nearly $3 \mathrm{~cm}$ would be required for $W_{\max }=200 \mu \mathrm{m}$ and $\lambda_{s}=1.059 \mu \mathrm{m}$. However, the first experimental demonstration of tapered structures in an ion-exchanged waveguide laser used a linear design with a faster expansion rate $(\alpha=1.75)$, opening to $175 \mu \mathrm{m}$ in just $12.5 \mathrm{~mm}$, while still delivering adiabatic operation [1]. Thus, in this paper we will experimentally compare the laser operation of linear and parabolic tapered waveguides, expanding to various widths over the same lengths, in order to work toward an optimized design for a high-power diode-pumped laser device. A fast expansion rate of $\alpha=2$ is chosen for this comparison, as it is beneficial to keep the device length as short as possible to minimize the propagation losses, and because it has already been shown that linear tapers can be successfully operated in this regime [1]. This comparison is followed by an initial demonstration of diode-pumped laser operation using a 2-W broad-stripe diode laser.

\section{TAPEREd WAVEguide Design AND FABRication}

In order to investigate the limitations of parabolic and linear-shaped tapers experimentally, we fabricated several sets of each kind of taper, alongside standard channel waveguides, on the same substrate. A 1.5-wt. $\% \mathrm{Nd}_{2} \mathrm{O}_{3}$-doped BK7 borosilicate glass slab had one large face polished in preparation for the ion exchange process. A 250-nm-thick aluminum film was deposited on the polished surface and standard photolithographic techniques were used to create openings in the film corresponding to the various taper designs and to simple $2.5-\mu \mathrm{m}$-wide channels of the same length. The waveguides 




Fig. 1. Design diagram for the parabolic and linear tapered waveguide structures.

TABLE I

DESIGN PARAMETERS FOR THE LiNEAR AND PARABOLIC TAPERED WAVEGUIDES

\begin{tabular}{ccc}
\hline$W_{\text {max }} / \mu \mathrm{m}$ & $l_{\text {tap }} / \mathrm{mm}$ & Common Parameters \\
\hline 175 & 11 & $n=1.51589$ \\
200 & 14.3 & $W_{0}=2.5 \mu \mathrm{m}$ \\
250 & 22.3 & $\lambda=1.059 \mu \mathrm{m}$ \\
& & $\alpha=2$ \\
\hline
\end{tabular}

were then formed by immersion in molten potassium nitrate at $395^{\circ} \mathrm{C}$ for $12 \mathrm{~h}$. Finally, the substrate was end-polished to yield plane end-faces at $90^{\circ}$ to the waveguide axes.

The taper mask was fabricated according to the designs given in Fig. 1 and using (3) and (4) for the parabolic and linearly tapered sections, respectively. The design is such that the linear and parabolic tapers expand to the same maximum widths over the same lengths. These equations satisfy the condition given by (2) for $W_{\max } \gg W_{0}$

$$
\begin{aligned}
W_{p}(z) & =\sqrt{\frac{2 \alpha \lambda_{s} z}{n}+W_{0}^{2}} \\
W_{l}(z) & =\frac{2 \alpha \lambda_{s}\left(W_{\max }-W_{0}\right) z}{n W_{\max }^{2}}+W_{0} .
\end{aligned}
$$

The resulting waveguide parameters are then listed in Table I for an expansion coefficient of $\alpha=2$. The total device length was kept constant at $46 \mathrm{~mm}$ by allowing the length of the single-mode channel section to vary from 3.7 to $15 \mathrm{~mm}$. It should be noted that the width of the ion-exchanged waveguide will be somewhat larger than the mask width due to lateral diffusion [5], but the greatest percentage effect will be on the width of the single-mode channel such that the overall expansion rate of the physical guide is slightly reduced.

\section{LASER Performance Characterization}

The laser performance of the tapers and channels was investigated by pumping the channel end of the waveguide with a Ti:sapphire laser tuned to the $\mathrm{Nd}^{3+}$ absorption at $807 \mathrm{~nm}$, as shown schematically in Fig. 2. The laser resonator was formed by butting thin mirrors to the plane end-faces of the waveguide substrate, using a thin film of fluorinated liquid to aid adhesion. The input mirror was highly reflecting at the lasing wavelength of $1.059 \mu \mathrm{m}$ and had $95 \%$ transmission at the pump wavelength, while the output coupler had a reflectivity $R_{o c}$ of $77 \%$ at the lasing wavelength. Figs. 3 and 4 show the laser results obtained for the laser threshold and slope efficiency with respect to absorbed pump power. It can be seen that, within experimental error, the slope efficiency and threshold of all the linear tapers is the same and is only slightly inferior to that of the channel guide, indicating that there is just a small increase in propagation loss due to the taper for the maximum taper widths and expansion rate investigated here. In contrast, it can be seen that the performance of the parabolic tapers is comparable to that of the linear tapers and channel guide for the $175-\mu \mathrm{m}$-wide taper, but the performance gets considerably worse for the larger widths.

The theoretical expectation for the slope efficiency with respect to absorbed power is given by [7]

$$
\eta_{\max }=\frac{\lambda_{p}}{\lambda_{s}}\left(\frac{-\ln R_{o c}}{-\ln R_{o c}+L}\right) \eta_{o l}
$$

where $L$ is the round-trip loss exponent and $\eta_{o l}$ is a factor accounting for the spatial overlap of the pump and signal radiation. We can express the roundtrip loss in $\mathrm{dB}$ as

$$
L_{\mathrm{dB}}=-10 \log _{10}\left(e^{-L}\right)=2 \delta l_{\mathrm{tot}}+\gamma
$$

where $\delta$ is the background propagation loss coefficient (assumed to be constant for all the waveguides), $l_{\text {tot }}$ is the total device length, and $\gamma$ is the additional (round-trip) loss due to the taper. Using this equation and assuming $\eta_{o l}=1$, a maximum possible propagation loss coefficient of $\delta=0.25 \mathrm{~dB} / \mathrm{cm}$ is obtained for the standard channel waveguide laser, which is a typical value for this type of waveguide. The assumption of a near unity overlap is reasonable for a single-mode waveguide where the pump spot size is always smaller than the laser spot size [7]. The additional loss due to the taper can then be calculated as $\gamma \leq 1.4 \mathrm{~dB}$ for the linear guides, whereas the additional loss for the parabolic taper rises to over $3 \mathrm{~dB}$ for the $200-\mu \mathrm{m}$-wide guide and over $10 \mathrm{~dB}$ for the $250-\mu \mathrm{m}$-wide guide. The low loss of the linear guides indicates an adiabatic expansion of the fundamental mode in the taper, and this was confirmed by the observation of clean Gaussian output profile on the charge-coupled device camera. In comparison, the output spatial profiles of the larger parabolic tapers display a distinctly multimode nature. The reason for the superior performance of the linear tapers is not clear. However, it should be noted that it has been suggested [6] that the high value of $\theta_{p}$ at the narrow end of the parabolic taper, a region where the propagating mode is not so strongly confined in the waveguide, may cause problems, and the design could be modified in this region to keep lower values of $\theta_{p}$ without significantly lengthening the taper. 


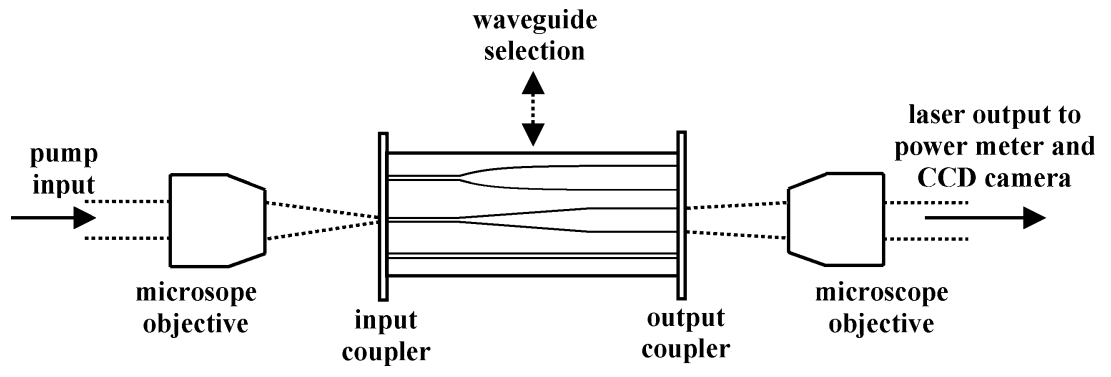

Fig. 2. Schematic representation of the experimental apparatus used to characterize the laser performance of the standard channel and tapered waveguide lasers.

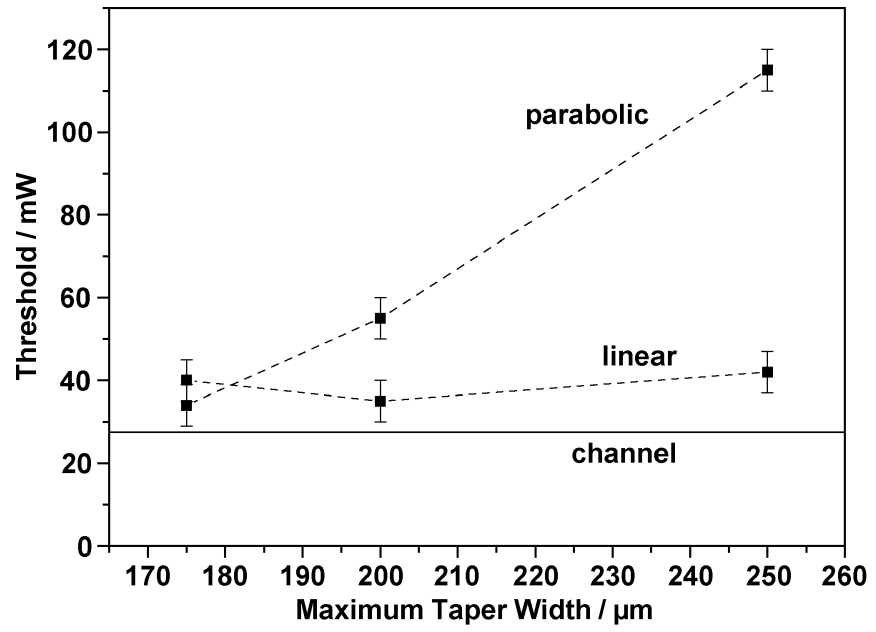

Fig. 3. Threshold absorbed pump power against maximum taper width for the linear and parabolic tapered waveguide lasers. The solid horizontal line represents the threshold for the standard channel waveguide lasers.

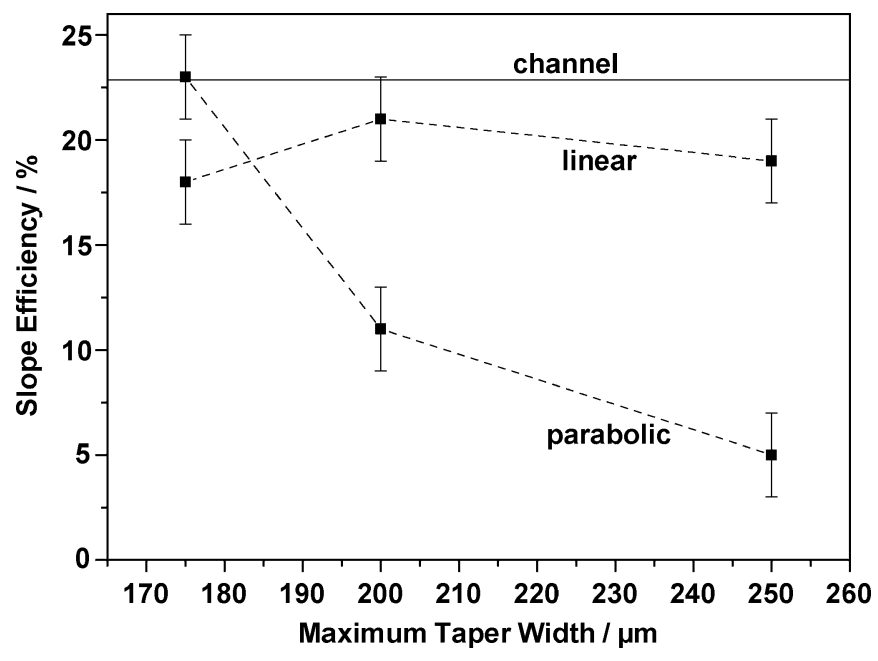

Fig. 4. Slope efficiency with respect to absorbed power against maximum taper width for the linear and parabolic waveguide lasers. The solid horizontal line represents the slope efficiency for the standard channel waveguide lasers.

\section{Diode-Pumped Laser Performance}

Initial results for diode-pumped operation have been obtained using a 2-W broad-stripe diode pump laser with a $1 \times 100 \mu \mathrm{m}^{2}$ emission area and $M^{2}$ values of 1.9 by 22 (measured after a collimation of the fast axis by a fiber lens). Two cylindrical lenses of focal lengths $19 \mathrm{~mm}$ (slow axis) and $12.7 \mathrm{~mm}$ (fast axis) were used to focus the beam, as shown in Fig. 5. The launch efficiency was measured in an undoped planar guide and an undoped $175-\mu \mathrm{m}$-wide broad-stripe guide, both fabricated under the same conditions as those described earlier, in order to assess the effect of the two guiding axes. Values of $67 \%$ (planar) and 54\% (broad-stripe) were found indicating that the ion-exchanged tapers of width $\geq 175 \mu \mathrm{m}$ are indeed compatible with broad-stripe pumping. It is believed that these figures could be optimized further if the focusing system was developed further and broader waveguides were used. The absorption of the Nd-doped bulk glass was measured using a similar broad-stripe diode source and a maximum absorption coefficient of $0.9 \mathrm{~cm}^{-1}$ was obtained. With these figures in mind an ion-exchanged linear tapered waveguide of maximum width $175 \mu \mathrm{m}$, taper length $12.5 \mathrm{~mm}$, broad-stripe length $25 \mathrm{~mm}$, and channel length $6 \mathrm{~mm}$ was fabricated in the Nd-doped glass substrate. For comparison, a $175-\mu \mathrm{m}$-wide broad-stripe waveguide of length $18 \mathrm{~mm}$ was also fabricated in a separate Nd-doped glass substrate. Using the same resonator as described earlier, laser action was obtained with a slope efficiency with respect to absorbed power $\eta$ of $27.5 \%$ for the broad-stripe waveguide and $12.9 \%$ for the tapered waveguide. The $M^{2}$ of the output of the broad-stripe waveguide laser was measured as 2 by 17, whereas the output of the tapered waveguide laser was diffraction-limited as it is extracted from the single-mode channel.

In Table II, we compare the laser performances of the twodiode-pumped lasers with the Ti:sapphire-pumped lasers described earlier. One possible reason why the slope efficiency would be lower in the diode-pumped taper compared to the similar Ti:sapphire-pumped tapers and standard channel guides (where we obtained $\sim 20 \%$ ) could be a worse spatial overlap between the pump and laser, in accordance with (5) and as calculated in Table II. This could arise from the fact that in the diode-pumped lasers this overlap is occurring in the multimode broad-stripe section of the taper rather than the single-mode channel section of the taper, as was the case for Ti:sapphire pumping. It should be noted that the broad-stripe sections of the waveguides are multimode in the depth axis as well as in the width axis, as evidenced by the multimode output of the broad-stripe waveguide laser, due to the fact that the thermal ion-exchange process leads to deeper guides for wider mask openings [5]. There is also an indication that this overlap may be worse in the diode-pumped taper, where a single-mode laser overlaps with a multimode pump, than in the broad-stripe laser, where both the laser and the pump are multimode. A possible future improvement to this design would be to use a more step-like 


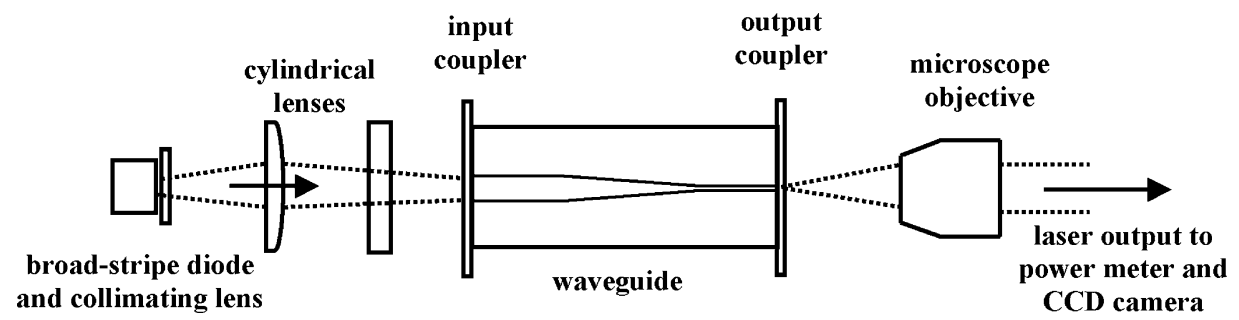

Fig. 5. Schematic representation of the experimental arrangement for the diode-pumped tapered waveguide laser.

TABLE II



\begin{tabular}{ccccc}
\hline & $\begin{array}{c}\text { Diode-Pumped } \\
\text { Broad-Stripe }\end{array}$ & $\begin{array}{c}\text { Diode-Pumped } \\
\text { Taper }\end{array}$ & $\begin{array}{c}\text { Ti:Sapphire- } \\
\text { Pumped Taper }\end{array}$ & Ti:Sapphire- \\
\hline$l_{\text {tot }} / \mathrm{mm}$ & 18 & 43.5 & 46 & 46 \\
$W_{\text {max }} / \mu \mathrm{m}$ & 175 & 175 & 175 & 2.5 \\
$\delta / \mathrm{dBcm}^{-1}$ & 0.25 & 0.25 & 0.25 & 0.25 \\
$\gamma / \mathrm{dB}$ & 0 & $0-1.4$ & 1.4 & 0 \\
$L^{2}$ & 0.207 & $0.501-0.823$ & 0.844 & 0.530 \\
$\eta($ measured $)$ & 0.275 & 0.129 & 0.18 & 0.23 \\
$\eta_{\text {ol }}($ calculated) & 0.65 & $0.49-0.70$ & 1 (assumed) & 1 (assumed) \\
Pump & Multimode & Multimode & Single Mode & Single Mode \\
Output & Multimode & Single Mode & Single Mode & Single Mode \\
\hline
\end{tabular}

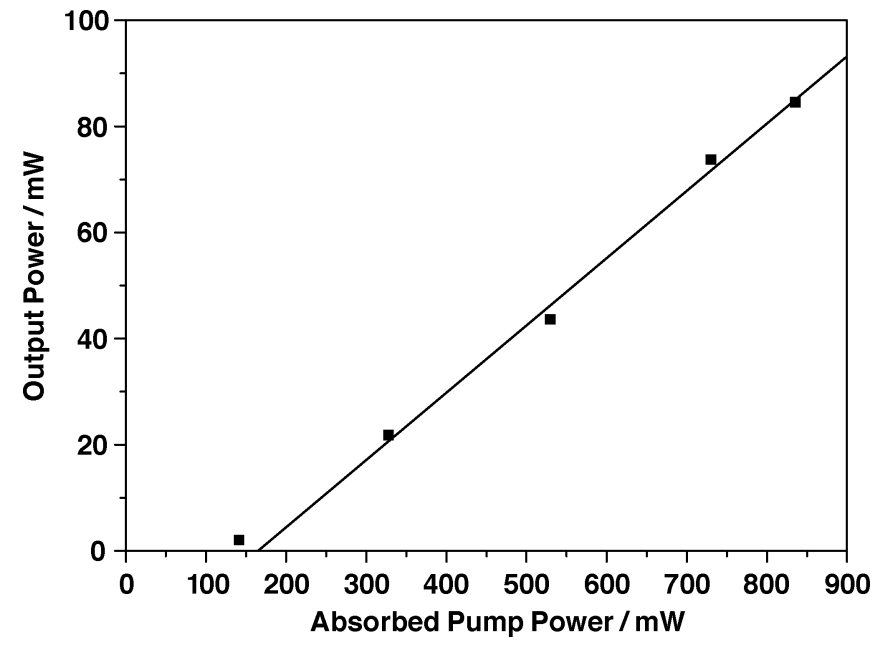

Fig. 6. Output power against absorbed pump power for the diode-pumped tapered waveguide laser.

index profile guide, for instance by using a field-assisted ion-exchange process, as such guides have a better overlap between the fundamental and higher order modes.

Fig. 6 shows the output power against absorbed pump power for the diode-pumped tapered waveguide laser. From the char- acterization provided here we can suggest various strategies for improving the output power of these integrated-optics laser sources. These include using linear tapers of increased width to improve the diode-launch efficiency and reduced length to reduce the background propagation losses. This can be done as long as the additional loss due to the taper does not significantly increase; a limit that has not yet been found with the 250- $\mu \mathrm{m}$-wide $\alpha=2$ linear tapers investigated here. Increasing the spatial overlap of the single-mode laser with the multimode pump could be possible by using a more step-like refractive index profile. Finally, higher power diode-pump lasers $(\sim 4 \mathrm{~W})$ of similar spatial quality are commercially available and would also allow the use of a bigger output-coupling fraction. It is estimated from our results that these improvements could lead to output powers $>0.5 \mathrm{~W}$.

\section{SUMMARY}

The performance of linear and parabolic tapers with fast expansion rates $(\alpha=2)$ to widths of up to $250 \mu \mathrm{m}$ has been compared. The linear tapers show just a small additional loss $(\sim 1$ $\mathrm{dB}$ ) over the background propagation loss of the ion-exchanged waveguide for the range of maximum taper widths investigated here, whereas parabolic tapers of the same length show a signif- 
icant reduction in performance for the larger widths. The large losses found for the parabolic tapered waveguide laser resonator were confirmed by the qualitative observation of higher order modes in the spatial profile of the output from the broad-stripe region. These results suggest that linear tapers are compatible with high-average-power pumping by broad-stripe diode lasers and should allow integrated lasers with powers of several hundred milliwatts. An initial demonstration of broad-stripe diodepumped operation has been reported at output power levels near $100 \mathrm{~mW}$, and an indication of the important parameters values required to optimize the output power has been given.

\section{ACKNOWLEDGMENT}

The authors would like to acknowledge Dr. J. I. Mackenzie for useful discussions and help on the diode-coupling scheme.

\section{REFERENCES}

[1] S. J. Hettrick, J. I. Mackenzie, R. D. Harris, J. S. Wilkinson, D. P. Shepherd, and A. C. Tropper, "Ion-exchanged tapered-waveguide laser in neodymium-doped BK7 glass," Opt. Lett., vol. 25, pp. 1433-1435, 2000.

[2] P. Madasamy, S. Honkanen, D. F. Geraghty, and N. Peyghambarian, "Single-mode tapered waveguide laser in Er-doped glass with multimode-diode pumping," Appl. Phys. Lett., vol. 82, pp. 1332-1334, 2003.

[3] J. D. Minelly, L. A. Zenteno, M. J. Dejneka, W. J. Miller, D. V. Kukzenkov, M. K. Davis, S. G. Crigler, and M. E. Bardo, "High power diode pumped single-transverse-mode $\mathrm{Yb}$ fiber laser operating at 978 nm," in Dig. Optical Fiber Communications Conf., 2000, postdeadline paper PD2.

[4] D. F. Welch, P. S. Cross, D. R. Scifres, W. Streifer, and R. D. Burnham, "High power, AlGaAs buried heterostructures lasers with flared waveguides," Appl. Phys. Lett., vol. 50, pp. 233-235, 1987.
[5] M. N. Weiss and R. Srivastava, "Determination of ion-exchanged channel waveguide profile parameters by mode-index measurements," Appl. Opt., vol. 34, pp. 455-458, 1995.

[6] A. F. Milton and W. K. Burns, "Mode coupling in optical waveguide horns," IEEE J. Quantum Electron., vol. QE-13, pp. 828-835, Oct. 1977.

[7] W. A. Clarkson and D. C. Hanna, "Effects of transverse-mode profile on slope efficiency and relaxation oscillations in a longitudinally pumped laser," J. Mod. Opt., vol. 36, pp. 483-498, 1989.

S. J. Hettrick, photograph and biography not available at the time of publication.

J. Wang, photograph and biography not available at the time of publication.

C. Li, photograph and biography not available at the time of publication.

J. S. Wilkinson, photograph and biography not available at the time of publication.

D. P. Shepherd, photograph and biography not available at the time of publication. 